\title{
Efficacy of Diuron Along with Sequential Application of Herbicides for Weed Control in Cotton
}

\author{
N. Varsha, M. Madhavi*, T. Ramprakash and K. B. Suneetha Devi
}

Department of Agronomy, College of Agriculture, Prof. Jayashankar Telangana State Agriculture University, Rajendranagar, Hyderabad-030, Telangana, India

*Corresponding author

Keywords

Diuron, Polymulch, weed control, phytotoxicity

Article Info

Accepted:

25 May 2018

Available Online:

10 June 2018

\section{A B S T R A C T}

A field experiments was conducted at Professor Jayashankar Telangana State Agricultural University, Rajendranagar during kharif 2017 for the evaluation of dosage of diuron suitable in both red and black soils. The treatment consisted of test herbicides such as, diuron $80 \% \mathrm{WP}$ at $0.5 \mathrm{~kg} \mathrm{ha}^{-1}, 0.75 \mathrm{~kg} \mathrm{ha}^{-1}$ and $1.0 \mathrm{~kg} \mathrm{ha}^{-1}$ along with registered formulation of pendimethalin $38.7 \% \mathrm{CS}$ at $677 \mathrm{~g} \mathrm{ha}^{-1}$, intercropping with green manure crop, mechanical weeding thrice at 20,40,60 DAS and unweeded control. The experiment was laid out in a randomised block design replicated thrice. The weed flora of the experimental field in red soil was dominated by Cynodon dactylon, Rottboellia exaltata, Parthenium hysterophorus, Trianthema portulacastrum, and Commelina benghalensis. While in case of black soil predominant flora was Cynodon dactylon, Cyperus rotundus, Parthenium hysterophorus, Euphorbia geniculata, Tridax procumbens, Cyanotis cristata, Digera arvensis and Celosia argentia. The treatments, mechanical weeding thrice at 20, 40 and 60 DAS, polymulch treatments reduced the weed growth in both red and black soils. Among the herbicides, diuron at $1.0 \mathrm{~kg} \mathrm{ha}^{-1} \mathrm{fb}$ pyrithiobac sodium + quizalofop $\mathrm{p}$ ethyl in red soil and both diuron at $1.0 \mathrm{~kg} \mathrm{ha}^{-1}$ and $0.75 \mathrm{~kg} \mathrm{ha}^{-1}$ along with sequential application of herbicides reduced the weed count and also the weed dry matter. The kapas and stalk yields were higher with polymulch, mechanical thrice at 20,40,60 DAS, while among the herbicides $1.0 \mathrm{~kg} \mathrm{ha}^{-1}$ in red soil and $0.75 \mathrm{~kg} \mathrm{ha}^{-1}$ in black soil.

\section{Introduction}

Cotton as a crop as well as commodity has a unique place in the economy of India as it plays an important role in the agrarian and industrial activities of the nation, being grown in acreage of $11.76 \mathrm{M}$ ha, while the total production of cotton in India is 6.21 million bales (170 kg each) in 2015-16 against the production of 34.80 million bales in 2014-15. The highest production is in Gujarat with 9.7 million bales $(32.18 \%)$ and second is Maharashtra with 6.5 million bales $(21.56 \%)$ and third leading producer is Telangana with 3.86 million bales $(12.80 \%)$.

In Telangana, the total area under cotton is 17.73 lakh hectares in 2015-16 and the production is 37.3 lakh bales lint of $170 \mathrm{kgs}$ in 2015-16. The productivity of cotton in

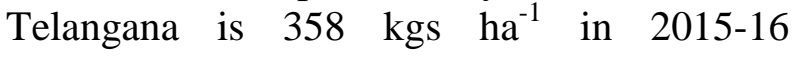
(Agriculture at a glance, 2016). 
Among different agronomic manipulations that would influence the productivity of cotton, management of unwanted plants called weeds is considered to be an important step for achieving higher productivity. Cotton, being a long duration, wide spaced and relatively slow growing crop in early stages, is subjected to a severe weed menace. Weeds primarily compete for nutrients, moisture and sunlight during the early crop growth period than at later stage. Weed infestation in cotton has been reported to offer severe competition and causing yield reduction to an extent of 74 per cent (Shelke and Bhosle, 1990), 50 to 85 per cent (Sharma, 2008) depending upon the nature and intensity of weeds. Every crop has a critical period of weed control which refers to the minimum time period during which the crop must be weed free. The critical period of weed competition in cotton was found to be 15 to 60 days (Sharma, 2008). Timely and effective weed management practices plays an important role in boosting the production of cotton. In India, manual and mechanical method of weed control continues to be the mainstay for the control of weeds. These methods are not only uneconomical, cumbersome, though they are more practically effective in controlling weeds. Usually weeding is done 3-4 times manually due to long period of crop growth. Hence, labour required for weeding is high, labour wages are increasing every year and non-availability of labour during peak period, resulting in increased cost of cultivation besides weedy condition. Manual weeding has traditionally been a labour intensive operation and hence there is no other alternative rather than use of post-emergence herbicides for control of existing weeds in cotton. Diuron has a prolonged soil residual life (80-230 days) making it more suitable for cotton crop due its slow initial growth. However, in the research experiment conducted in PJTSAU, it was observed that diuron $80 \% \mathrm{WP}$ applied at 1.0 $\mathrm{kg} \mathrm{ha}{ }^{-1}$ caused significant reduction in plant stand in black soils which shows the need for the re-evaluation of herbicide dose in black and red soils also. At present, pendimethalin and alachlor are the two pre-emergence (PE) herbicides registered for use in cotton (other than diuron). As alachlor is being phased out of use by 2020, pendimethalin will be the only PE herbicide for cotton. As utilising the same herbicide over long period will result in poor bioefficacy and development of resistance in weeds, there is a need to evaluate the diuron for pre-emergence use, keeping in view the safe practice of rotating herbicides with different modes of action. Hence the study was conducted for the evaluation of weed management in cotton in red and black soils

\section{Materials and Methods}

A field experiment was conducted during kharif, 2017 at College farm, College of Agriculture, Professor Jayashankar Telangana State Agricultural University, Rajendranagar, Hyderabad, Telangana State. The farm is geographically located at an altitude of 542.3 $\mathrm{m}$ above mean sea level at $17^{\circ} 19^{\prime} \mathrm{N}$ latitude and $78^{\circ} 23^{\prime}$ E longitude in the Southern Telangana agro-climatic zone of Telangana. According to Troll's (Troll, 1958) climatic classification, it falls under semi-arid tropics (SAT). The experiment was conducted in red and black soil and laid out in a randomised block design with three replications. The treatments included three doses of diuron $(0.5$, 0.75 and $1.0 \mathrm{~kg} \mathrm{ha}^{-1}$ ), pendimethalin $38.7 \%$ $\mathrm{CS}$ at $677 \mathrm{~g} \mathrm{ha}^{-1}$ as PE followed by sequential application of pyrithiobac sodium 10\% EC $62.5 \mathrm{~g} \mathrm{ha}^{-1}+$ quizalofop p ethyl $5 \%$ EC $50 \mathrm{~g}$ $\mathrm{ha}^{-1}$, intercropping of cotton with green manure crop (sunhemp), mechanical weeding thrice at 20, 40 and 60 DAS (weed free), polymulch and unweeded control.

Mallika $B t$ was sown with a seed rate of $2.5 \mathrm{~kg}$ $\mathrm{ha}^{-1}$. One-two seeds per hill were sown at a spacing of $75 \mathrm{~cm} \mathrm{X} 75 \mathrm{~cm}$ to facilitate the use 
of power weeder in both directions in case of mechanical weeding. Pre-emergence herbicides were sprayed on the third day after sowing. Diuron $80 \%$ W.P. at $0.5 \mathrm{~kg} \mathrm{ha}^{-1}$, diuron $80 \%$ W.P. at $0.75 \mathrm{~kg} \mathrm{ha}^{-1}$, diuron $80 \%$ WP $1.0 \mathrm{~kg} \mathrm{ha}^{-1}$, pendimethalin $38.7 \%$ CS 677 $\mathrm{g} \mathrm{ha}^{-1}$ were sprayed on the third day, pyrithiobac sodium 10\% EC $62.5 \mathrm{~g} \mathrm{ha}^{-1}+$ quizalofop p ethyl 5\% EC $50 \mathrm{~g} \mathrm{ha}^{-1}$ were sprayed at 2-3 leaf stage of the weeds. In the intercropping treatment the intercrop sunhemp was sown along with cotton. Polymulch was spread 8 DAS after emergence of the seedling. Mechanical weeding at 20, 40, 60 DAS was done with power weeder and an unweeded check was maintained.

The important weed species associated with Bt Cotton crop in the experimental area were recorded at 30, 60 and 90 DAS. Weed count was taken at 30, 60 and 90 DAS in two randomly selected quadrats $(0.5 \mathrm{~m} \mathrm{x} 0.5 \mathrm{~m})$ in each plot. At every sampling, individual species were separated and expressed as number $\mathrm{m}^{-2}$. The sampling was done outside the net plot but within the gross plot.

The treatment wise total weed count was recorded and expressed as no. $\mathrm{m}^{-2}$. The total weeds enclosed in the quadrat were carefully cut close to the ground level with the help of weeding hook. All the weeds from each quadrat were collected, air dried and then oven dried at $65 \pm 5{ }^{\circ} \mathrm{C}$ temperature till the constant weight was obtained. Thereafter by averaging dry weight of weeds from two quadrats, treatment wise total dry matter of weed was recorded and expressed as $\mathrm{g} \mathrm{m}^{-2}$. At each picking, seed cotton obtained from the net plot was weighed. The cumulative yield from three pickings $(127,142$ and 167) in plots in each treatment was expressed as yield in $\mathrm{kg} \mathrm{ha}^{-1}$. The cotton stalk was uprooted from net plot area of treatment and sun dried for one week and the weight was recorded. The stalk yield $\left(\mathrm{kg} \mathrm{ha}^{-1}\right)$ was worked out.

\section{Results and Discussion}

\section{Weed Flora}

The study was conducted in two different soils i.e., red and black soils. The weed flora was observed in both the soils was recorded.

In the red soil, among the grasses Cynodon dactylon, Rottboellia exaltata, Dactyloctenium aegyptium and Dinebra retroflexa were noticed. Cyperus rotundus was the only sedge present in the field. Among the broad leaved weeds, Parthenium hysterophorus, Euphorbia geniculata, Trianthema portulacastrum, Trichodesma indica, Commelina benghalensis, Digera arvensis, Tridax procumbens, and Phyllanthus niruri were observed in the field.

In the black soil, among the grasses Cynodon dactylon, Rottboellia exaltata and Echinocloa colonum, Dactyloctenium aegyptium were noticed. Cyperus rotundus was the only sedge present in the field. Among the broad leaved weeds, Parthenium hysterophorus, Euphorbia geniculata, Trianthema portulacastrum, Trichodesma indica, Cyanotis cristata, Digera arvensis and Celosia argentia were observed in the field

\section{Total weed density}

The data regarding total weed density is presented in table 1.

\section{Red soil}

The perusal of the data at 30 DAS revealed that mechanical weeding thrice (8.67) and polymulch (10.67) treatments registered significantly lowest weed density and diuron $1.0 \mathrm{~kg} \mathrm{ha}^{-1}$ as PE $f b$ pyrithiobac sodium + quizalofop p ethyl as PoE (20.00) was on par polymulch treatment. Diuron $0.75 \mathrm{~kg} \mathrm{ha}^{-1} \mathrm{PE}$ $f b$ pyrithiobac sodium + quizalofop $\mathrm{p}$ ethyl 
PoE (30.33) was statistically on par with diuron $1.0 \mathrm{~kg} \mathrm{ha}^{-1}$ as $\mathrm{PE} f b$ pyrithiobac sodium + quizalofop $\mathrm{p}$ ethyl as PoE which were significantly superior among the herbicides. The treatment intercropping with sunhemp (53.00) also registered higher weed density which was on par with unweeded control (63.67) and recorded the significantly higher weed density (63.67).

At 60 DAS, polymulch (17.00) and mechanical weeding thrice (18.00) treatments registered significantly lower weed density. Among the herbicides diuron $1.0 \mathrm{~kg} \mathrm{ha}^{-1}$ as PE $f b$ pyrithiobac sodium + quizalofop $\mathrm{p}$ ethyl as PoE (41.33) was on par with the mechanical weeding thrice treatment and also it was significantly superior to diuron $0.75 \mathrm{~kg} \mathrm{ha}^{-1}$ or $0.5 \mathrm{~kg} \mathrm{ha}^{-1}$ as PE $f b$ pyrithiobac sodium + quizalofop $\mathrm{p}$ ethyl as POE. Intercropping of cotton with sunhemp also registered comparatively lower weed density as the sunhemp was cut and spread as mulch. The unweeded control recorded the highest weed density among all the treatments (75.00). At 90 DAS, the weed density was greatly reduced due to the crop growth which covered the ground area and suppressed the weed growth. Mechanical weeding thrice (10.33) and polymulch (10.33) treatments registered lowest weed density which were significantly superior to the rest of the treatments. Among the herbicides, diuron at $1.0 \mathrm{~kg} \mathrm{ha}^{-1}$ as $\mathrm{PE} f b$ pyrithiobac sodium + quizalofop $\mathrm{p}$ ethyl as PoE (21.33) and diuron $0.75 \mathrm{~kg} \mathrm{ha}^{-1} \mathrm{PE} f b$ pyrithiobac sodium + quizalofop $\mathrm{p}$ ethyl $\mathrm{PoE}$ (24.67) were on par and significantly superior over other treatments. As usual, the unweeded control recorded the highest weed density among all the treatments (59.67).

\section{Black soil}

At 30 DAS, the minimum weed density was recorded in mechanical weeding thrice at 20 , 40, 60 DAS (17.67), polymulch (18.00) which were significantly superior over other treatments. Diuron $1.0 \mathrm{~kg} \mathrm{ha}^{-1}$ as PE $f b$ pyrithiobac sodium + quizalofop $\mathrm{p}$ ethyl as POE and diuron $0.75 \mathrm{~kg} \mathrm{ha}^{-1}$ as PE $f b$ pyrithiobac sodium + quizalofop $\mathrm{p}$ ethyl as POE which were on par with each other The significantly higher weed density was reported in unweeded control (114.00) which was on par with intercropping with green manure (101.33). At 60 DAS, polymulch (11.33) treatment registered minimum weed density which was significantly superior in weed control over other treatments which was $f b$ mechanical weeding thrice. Diuron $1.0 \mathrm{~kg} \mathrm{ha}^{-1}$ as PE $f b$ pyrithiobac sodium + quizalofop $\mathrm{p}$ ethyl as PoE, diuron $0.75 \mathrm{~kg} \mathrm{ha}^{-1}$ as PE $f b$ pyrithiobac sodium + quizalofop p ethyl as PoE, pendimethalin as $\mathrm{PE} f b$ pyrithiobac sodium + quizalofop $\mathrm{p}$ ethyl as POE, diuron $80 \% \mathrm{WP}$ at $0.5 \mathrm{~kg} \mathrm{ha}^{-1}$ as PE $f b$ pyrithiobac sodium + quizalofop $\mathrm{p}$ ethyl as POE and intercrop were on par which each other. The maximum weed number per $\mathrm{m}^{2}$ was observed in unweeded control (99.67) which was significantly higher compared to others. At 90 DAS, the least weed number per $\mathrm{m}^{2}$ was observed with mechanical weeding thrice (9.67) and polymulch (10.67) which were significantly superior over herbicides. Diuron $1.0 \mathrm{~kg} \mathrm{ha}^{-1}$ as PE $f b$ pyrithiobac sodium + quizalofop $\mathrm{p}$ ethyl as PoE, diuron $0.75 \mathrm{~kg} \mathrm{ha}^{-1}$ as PE $f b$ pyrithiobac sodium + quizalofop $\mathrm{p}$ ethyl as PoE, pendimethalin as PE $f b$ pyrithiobac sodium + quizalofop p ethyl as POE and intercrop were on par with each other. The maximum weed density was observed in unweeded control which was the significantly the highest (55.00).

The season long reduced density of weeds in polythene mulch might be due to the sensitivity of the most of the weed seeds to light. So these weed seeds did not germinate under the plastic mulch so ultimately caused a reduction in population (Mahajan et al., 2007). Sequential application of herbicides along 
with application of PoE herbicides resulted in lower weed density which could be attributed to weed free situation during initial stages and further control of new flush of weeds by application of post emergence herbicides at 25 DAS and thus, reducing the weed competition during critical initial to peak growth period of $B t$ cotton. Similar results were reported by Chetan (2016), Prabhu (2011), Nalini et al., (2013) and Hariharasudhan, (2017).

\section{Weed dry matter $\left(\mathrm{g} \mathrm{m}^{-2}\right)$}

As weed control practices are concerned, different weed control treatments also had significant effect on dry weight of weeds. The data regarding weed dry matter is presented in table 2 .

\section{Red soil}

At 30 DAS, mechanical weeding thrice (3.22 $\left.\mathrm{g} \mathrm{m}^{-2}\right)$ and polymulch $\left(3.93 \mathrm{~g} \mathrm{~m}^{-2}\right)$ treatments significantly reduced the weed dry matter and the diuron $1.0 \mathrm{~kg} \mathrm{ha}^{-1}$ as PE $f b$ pyrithiobac sodium + quizalofop p ethyl as PoE (3.90 $\mathrm{g} \mathrm{m}^{-}$ ${ }^{2}$ ) was statistically on par with the above treatments. On the other hand, diuron $0.75 \mathrm{~kg}$ $\mathrm{ha}^{-1}$ as PE $f b$ pyrithiobac sodium + quizalofop $p$ ethyl as PoE was on par with diuron $1.0 \mathrm{~kg}$ $\mathrm{ha}^{-1}$ and was significantly superior in controlling the dry weight of weeds. The treatment intercropping with sunhemp (15.63 $\mathrm{g} \mathrm{m}^{-2}$ ) did not reduce weed dry matter as neither herbicide nor weeding were taken and also the crop growth was slow to suppress the weeds and was on par with unweeded control $\left(19.34 \mathrm{~g} \mathrm{~m}^{-2}\right)$

Among the different weed control options adopted at 60 DAS, mechanical weeding thrice $\left(7.60 \mathrm{~g} \mathrm{~m}^{-2}\right)$ and polymulch $\left(8.30 \mathrm{~g} \mathrm{~m}^{-2}\right)$ treatments have significantly reduced the dry weight of weeds. Diuron $1.0 \mathrm{~kg} \mathrm{ha}^{-1}$ as PE $f b$ pyrithiobac sodium + quizalofop $\mathrm{p}$ ethyl as PoE $\left(17.70 \mathrm{~g} \mathrm{~m}^{-2}\right)$ significantly lowered the weed DMP than other herbicidal treatments. The unweeded control recorded the highest weed density among all the treatments $(54.70$ $\mathrm{m}^{-2}$ ). Similar results were also observed at 90 DAS. The Weed dry weight was significantly higher under unweeded control at all stages of observation.

\section{Black soil}

At 30 DAS, the minimum weed dry matter was observed in mechanical weeding thrice at 20, 40, 60 DAS $\left(6.45 \mathrm{~g} \mathrm{~m}^{-2}\right)$, polymulch $(6.56$ $\mathrm{g} \mathrm{m}^{-2}$ ) and diuron $1.0 \mathrm{~kg} \mathrm{ha}^{-1}$ as PE $f b$ pyrithiobac sodium + quizalofop $\mathrm{p}$ ethyl as PoE $\left(8.12 \mathrm{~g} \mathrm{~m}^{-2}\right)$ and were significantly superior in weed control. Diuron at $0.75 \mathrm{~kg} \mathrm{ha}^{-}$ ${ }^{1}$ as PE $f b$ pyrithiobac sodium + quizalofop $\mathrm{p}$ ethyl as PoE $\left(9.21 \mathrm{~g} \mathrm{~m}^{-2}\right)$ was on par with diuron $1.0 \mathrm{~kg} \mathrm{ha}^{-1}$ as PE $f b$ pyrithiobac sodium + quizalofop p ethyl as PoE. At 60 DAS, mechanical weeding thrice at 20,40,60 DAS $\left(18.08 \mathrm{~g} \mathrm{~m}^{-2}\right)$, polymulch $\left(6.56 \mathrm{~g} \mathrm{~m}^{-2}\right)$ registered significantly lower weed dry matter. Diuron $1.0 \mathrm{~kg} \mathrm{ha}^{-1}$ as PE $f b$ pyrithiobac sodium + quizalofop p ethyl as PoE (20.99 g $\mathrm{m}^{-2}$ ) was on par with that of polymulch treatment.

Diuron at $0.75 \mathrm{~kg} \mathrm{ha}^{-1}$ as $\mathrm{PE} f b$ pyrithiobac sodium + quizalofop p ethyl as PoE $(23.02 \mathrm{~g}$ $\mathrm{m}^{-2}$ ) was on par with diuron $1.0 \mathrm{~kg} \mathrm{ha}^{-1}$ as PE $f b$ pyrithiobac sodium + quizalofop $\mathrm{p}$ ethyl as PoE and were significantly superior in reducing the weed dry matter among the herbicides.

At 90 DAS, the least weed dry matter production was observed with mechanical weeding thrice $\left(15.31 \mathrm{~g} \mathrm{~m}^{-2}\right)$, polymulch $\left(15.56 \mathrm{~g} \mathrm{~m}^{-2}\right)$, diuron $1.0 \mathrm{~kg} \mathrm{ha}^{-1}$ as PE $f b$ pyrithiobac sodium + quizalofop p ethyl as PoE $\left(15.04 \mathrm{~g} \mathrm{~m}^{-2}\right)$ and diuron at $0.75 \mathrm{~kg} \mathrm{ha}^{-1}$ as $\mathrm{PE} f b$ pyrithiobac sodium + quizalofop $\mathrm{p}$ ethyl as PoE $\left(15.36 \mathrm{~g} \mathrm{~m}^{-2}\right)$ which were significantly superior in reducing the weed dry matter. 
The significant reduction in total weed dry weight might be due to reduced numbers of weeds available due to the influence of the sequential use of PE and PoE herbicides. Initial flush of weeds were controlled by preemergence herbicide while subsequent flush of weeds were controlled by the combined spray of grassy herbicide (quizalofop ethyl) and broad leaf herbicide (pyrithiobac sodium) at 25 DAS. These results are in accordance with Hargilas (2015) and Hiremath et al., (2013). The minimum weed dry matter was recorded in polymulch which can be ascribed to mulches which suppress the weeds growth mainly by restricting the light penetration into the soil. These results are in line with the findings of Ather et al., (2013).

\section{Weed control efficiency $(\%)$}

Weed control efficiency exhibited variation among different weed control treatments which is an index to reduction in weed dry matter at different growth stages. The data on WCE is presented in table 3.

\section{Red soil}

At all the stages of the crop growth, mechanical weeding thrice $(88.95 \%, 86.08 \%$ and $75.29 \%$ respectively at $30,60,90$ DAS) and polymulch $(85.79 \%, 84.93 \%$ and $73.50 \%$ respectively at $30,60,90 \mathrm{DAS})$ recorded the maximum WCE. Among the herbicidal treatments, diuron $1.0 \mathrm{~kg} \mathrm{ha}^{-1}$ as PE $f b$ pyrithiobac sodium + quizalofop $\mathrm{p}$ ethyl as POE $(79.49 \%, \quad 67.79 \%$ and $62.40 \%$ respectively at $30,60,90 \mathrm{DAS}$ ) had given a better weed control than chemical treatments and was $f b$ diuron $0.75 \mathrm{~kg} \mathrm{ha}^{-1}$ as PE $f b$ pyrithiobac sodium + quizalofop $p$ ethyl as PoE, diuron $0.5 \mathrm{~kg} \mathrm{ha}^{-1}$ as PE $f b$ pyrithiobac sodium + quizalofop $\mathrm{p}$ ethyl as $\mathrm{PoE}$ and pendimethalin as $\mathrm{PE} f b$ pyrithiobac sodium + quizalofop $\mathrm{p}$ ethyl as POE. While the least WCE was reported in the intercropping of cotton with green manure $(34.17 \%, 28.88 \%$ and $31.62 \%$ respectively at $30,60,90$ DAS).

\section{Black soil}

Similar to the results in red soils, even in the black soils mechanical weeding thrice $(94.86 \%, 82.88 \%$ and $65.12 \%$ respectively at $30,60,90$ DAS) and polymulch $(94.65 \%$, $79.77 \%$ and $65.12 \%$ respectively at $30,60,90$ DAS) recorded the highest WCE. Among the herbicides, diuron $1.0 \mathrm{~kg} \mathrm{ha}^{-1}$ as $\mathrm{PE} f b$ pyrithiobac sodium + quizalofop $\mathrm{p}$ ethyl as PoE (91.40\%, $77.27 \%$ and $66.06 \%$ respectively at $30,60,90 \mathrm{DAS}$ ) had given a superior weed control and was closely $f b$ diuron $0.75 \mathrm{~kg} \mathrm{ha}^{-1}$ as $\mathrm{PE} f b$ pyrithiobac sodium + quizalofop $\mathrm{p}$ ethyl as PoE. Diuron $0.5 \mathrm{~kg} \mathrm{ha}^{-1}$ as PE $f b$ pyrithiobac sodium + quizalofop $\mathrm{p}$ ethyl as $\mathrm{PoE}$ and pendimethalin as $\mathrm{PE} f b$ pyrithiobac sodium + quizalofop $\mathrm{p}$ ethyl as PoE recorded lower WCE compared to higher doses of diuron. However the least WCE was reported in the intercropping of cotton with sunhemp at all the stages of crop growth. Polymulch could effectively suppress the germination and growth of weeds which gave an upper hand to the crop.

Similar findings were reported by Hiremath et al., (2013) and Prabhu, (2010) who stated that highest weed control efficiency was recorded with diuron $80 \mathrm{WP}$ at $1 \mathrm{~kg} \mathrm{ha}^{-1}$ and pendimethalin $38.7 \mathrm{CS}$ at $0.68 \mathrm{~kg} \mathrm{ha}^{-1}$ as diuron helps the crop to establish better and make early growth under weed free situation and pendimethalin also had similar effects.

\section{Weed index $(\%)$}

Weed index is a calculated using the yield obtained in the treated plot and weed free plot which provides information reduction in crop yield due to presence of weeds in comparison with weed free treatment and thus the effectiveness of the treatment can be judged. 
Table.1 Total weed density (no $\mathrm{m}^{-2}$ ) as influenced by weed control options in cotton

\begin{tabular}{|c|c|c|c|c|c|c|}
\hline \multirow[t]{2}{*}{ TREATMENTS } & \multicolumn{3}{|c|}{ Red soil } & \multicolumn{3}{|c|}{ Black soil } \\
\hline & 30 DAS & 60 DAS & 30 DAS & 30 DAS & 60 DAS & 90 DAS \\
\hline $\begin{array}{l}\text { Diuron } 80 \% \text { WP } 0.5 \mathrm{~kg} \mathrm{ha}^{-1} \text { fb pyrithiobac sodium } \\
10 \% \text { EC } 62.5 \mathrm{~g} \mathrm{ha}^{-1}+\text { quizalofop p ethyl } 5 \% \text { EC } 50 \mathrm{~g} \\
\mathrm{ha}^{-1}\end{array}$ & $\begin{array}{c}5.97 \\
(35.00)\end{array}$ & $\begin{array}{c}8.02 \\
(63.67)\end{array}$ & $\begin{array}{c}5.97 \\
(35.00)\end{array}$ & $\begin{array}{c}8.24 \\
(67.33)\end{array}$ & $\begin{array}{c}8.02 \\
(63.33)\end{array}$ & $\begin{array}{c}7.01 \\
(49.00)\end{array}$ \\
\hline $\begin{array}{l}\text { Diuron } 80 \% \text { WP } 0.75 \mathrm{~kg} \mathrm{ha}^{-1} \text { fb pyrithiobac sodium } \\
10 \% \text { EC } 62.5 \mathrm{~g} \mathrm{ha}^{-1}+\text { quizalofop p ethyl } 5 \% \text { EC } 50 \mathrm{~g} \\
\text { ha }^{-1}\end{array}$ & $\begin{array}{c}5.59 \\
(30.33)\end{array}$ & $\begin{array}{c}7.93 \\
(62.00)\end{array}$ & $\begin{array}{c}5.59 \\
(30.33)\end{array}$ & $\begin{array}{c}6.22 \\
(38.00)\end{array}$ & $\begin{array}{c}7.91 \\
(61.67)\end{array}$ & $\begin{array}{c}5.65 \\
(31.00)\end{array}$ \\
\hline $\begin{array}{l}\text { Diuron } 80 \% \text { WP } 1.0 \mathrm{~kg} \mathrm{ha}^{-1} \text { fb pyrithiobac sodium } \\
10 \% \text { EC } 62.5 \mathrm{~g} \mathrm{ha}^{-1}+\text { quizalofop p ethyl } 5 \% \text { EC } 50 \mathrm{~g} \\
\mathrm{ha}^{-1}\end{array}$ & $\begin{array}{c}4.57 \\
(20.00)\end{array}$ & $\begin{array}{c}6.50 \\
(41.33)\end{array}$ & $\begin{array}{c}4.57 \\
(20.00)\end{array}$ & $\begin{array}{c}5.84 \\
(34.00)\end{array}$ & $\begin{array}{c}7.28 \\
(52.00)\end{array}$ & $\begin{array}{c}5.57 \\
(30.00)\end{array}$ \\
\hline $\begin{array}{l}\text { Pendimethalin } 38.7 \% \text { CS at } 677 \mathrm{~g} \mathrm{ha}^{-1} \text { fb pyrithiobac } \\
\text { sodium } 10 \% \text { EC } 62.5 \mathrm{~g} \mathrm{ha}^{-1}+\text { quizalofop p ethyl } 5 \% \\
\text { EC } 50 \mathrm{~g} \mathrm{ha}^{-1}\end{array}$ & $\begin{array}{c}6.98 \\
(49.00)\end{array}$ & $\begin{array}{c}7.76 \\
(59.33)\end{array}$ & $\begin{array}{c}6.98 \\
(49.00)\end{array}$ & $\begin{array}{c}8.84 \\
(77.33)\end{array}$ & $\begin{array}{c}7.86 \\
(61.00)\end{array}$ & $\begin{array}{c}6.14 \\
(38.00)\end{array}$ \\
\hline $\begin{array}{l}\text { Cotton + sunhemp (sunhemp was cut and spread as } \\
\text { mulch) }\end{array}$ & $\begin{array}{c}7.34 \\
(53.00)\end{array}$ & $\begin{array}{c}6.31 \\
(39.67)\end{array}$ & $\begin{array}{c}7.34 \\
(53.00)\end{array}$ & $\begin{array}{c}10.12 \\
(101.33)\end{array}$ & $\begin{array}{c}7.70 \\
(58.33)\end{array}$ & $\begin{array}{c}6.31 \\
(39.00)\end{array}$ \\
\hline Mechanical weeding at 20, 40, 60 DAS (weed free) & $\begin{array}{c}3.06 \\
(8.67)\end{array}$ & $\begin{array}{c}4.35 \\
(18.00)\end{array}$ & $\begin{array}{c}3.06 \\
(8.67)\end{array}$ & $\begin{array}{c}4.28 \\
(17.67)\end{array}$ & $\begin{array}{c}4.76 \\
(23.00)\end{array}$ & $\begin{array}{l}3.26 \\
(9.67)\end{array}$ \\
\hline Control (unweeded) & $\begin{array}{c}8.04 \\
(63.67)\end{array}$ & $\begin{array}{c}8.71 \\
(75.00)\end{array}$ & $\begin{array}{c}8.04 \\
(63.67)\end{array}$ & $\begin{array}{c}10.72 \\
(114.00)\end{array}$ & $\begin{array}{c}9.98 \\
(99.67)\end{array}$ & $\begin{array}{c}7.43 \\
(55.00)\end{array}$ \\
\hline Polymulch of $0.25 \mathrm{~mm}$ thickness & $\begin{array}{c}3.40 \\
(10.67)\end{array}$ & $\begin{array}{c}4.21 \\
(17.00)\end{array}$ & $\begin{array}{c}3.40 \\
(10.67)\end{array}$ & $\begin{array}{c}4.34 \\
(18.00)\end{array}$ & $\begin{array}{c}3.49 \\
(11.33)\end{array}$ & $\begin{array}{c}3.41 \\
(10.67)\end{array}$ \\
\hline $\mathrm{SE}(\mathrm{m}) \pm$ & 0.416 & 0.241 & 0.416 & 0.369 & 0.405 & 0.347 \\
\hline C.D. $(p=0.05)$ & 1.275 & 0.737 & 0.780 & 1.130 & 1.240 & 1.062 \\
\hline
\end{tabular}

Figures in parenthesis are original values and data is subjected $\sqrt{X+1}$ transformation 
Table. 2 Weed dry matter $\left(\mathrm{g} \mathrm{m}^{-2}\right)$ as influenced by weed control options in cotton

\begin{tabular}{|c|c|c|c|c|c|c|}
\hline \multirow{2}{*}{ TREATMENTS } & \multicolumn{3}{|c|}{ Red soil } & \multicolumn{3}{|c|}{ Black soil } \\
\hline & 30 DAS & 60 DAS & 90 DAS & 30 DAS & 60 DAS & 90 DAS \\
\hline 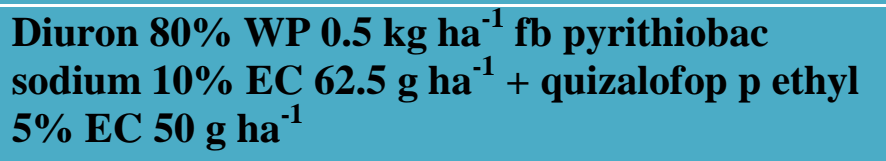 & $\begin{array}{c}3.56 \\
(11.76)\end{array}$ & $\begin{array}{c}5.69 \\
(31.50)\end{array}$ & $\begin{array}{c}5.14 \\
(25.40)\end{array}$ & $\begin{array}{c}4.23 \\
(16.92)\end{array}$ & $\begin{array}{c}5.88 \\
(33.57)\end{array}$ & $\begin{array}{c}4.56 \\
(19.79)\end{array}$ \\
\hline 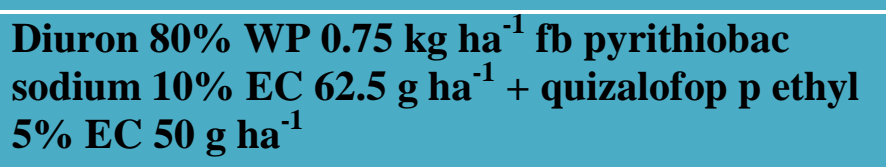 & $\begin{array}{c}2.66 \\
(6.16)\end{array}$ & $\begin{array}{c}5.14 \\
(25.50)\end{array}$ & $\begin{array}{c}4.34 \\
(17.90)\end{array}$ & $\begin{array}{c}3.18 \\
(9.21)\end{array}$ & $\begin{array}{c}4.90 \\
(23.02)\end{array}$ & $\begin{array}{c}4.07 \\
(15.56)\end{array}$ \\
\hline 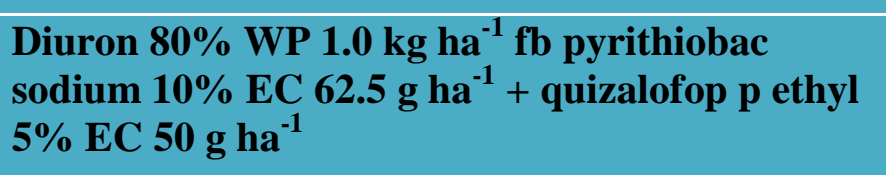 & $\begin{array}{c}2.21 \\
(3.90)\end{array}$ & $\begin{array}{c}4.31 \\
(17.70)\end{array}$ & $\begin{array}{c}3.98 \\
(14.90)\end{array}$ & $\begin{array}{c}3.02 \\
(8.12)\end{array}$ & $\begin{array}{c}4.69 \\
(20.99)\end{array}$ & $\begin{array}{c}4.04 \\
(15.36)\end{array}$ \\
\hline $\begin{array}{l}\text { Pendimethalin } 38.7 \% \text { CS at } 677 \mathrm{~g} \mathrm{ha}^{-1} \mathrm{fb} \\
\text { pyrithiobac sodium } 10 \% \mathrm{EC} 62.5 \mathrm{~g} \mathrm{ha}^{-1}+ \\
\text { quizalofop p ethyl } 5 \% \mathrm{EC} 50 \mathrm{~g} \mathrm{ha}^{-1}\end{array}$ & $\begin{array}{c}3.76 \\
(13.20)\end{array}$ & $\begin{array}{c}5.81 \\
(32.90)\end{array}$ & $\begin{array}{c}4.99 \\
(23.90)\end{array}$ & $\begin{array}{c}4.21 \\
(16.77)\end{array}$ & $\begin{array}{c}5.96 \\
(34.50)\end{array}$ & $\begin{array}{c}4.73 \\
(21.37)\end{array}$ \\
\hline $\begin{array}{l}\text { Cotton }+ \text { sunhemp (sunhemp was cut and spread } \\
\text { as mulch) }\end{array}$ & $\begin{array}{c}4.06 \\
(15.63)\end{array}$ & $\begin{array}{c}6.31 \\
(38.90)\end{array}$ & $\begin{array}{c}5.29 \\
(27.05)\end{array}$ & $\begin{array}{c}4.53 \\
(19.55)\end{array}$ & $\begin{array}{c}5.51 \\
(29.37)\end{array}$ & $\begin{array}{c}4.82 \\
(22.27)\end{array}$ \\
\hline $\begin{array}{l}\text { Mechanical weeding at 20, 40, } 60 \text { DAS (weed } \\
\text { free) }\end{array}$ & $\begin{array}{c}2.04 \\
(3.22)\end{array}$ & $\begin{array}{c}2.92 \\
(7.60)\end{array}$ & $\begin{array}{c}3.27 \\
(9.75)\end{array}$ & $\begin{array}{c}2.73 \\
(6.45)\end{array}$ & $\begin{array}{c}4.36 \\
(18.08)\end{array}$ & $\begin{array}{c}4.00 \\
(15.04)\end{array}$ \\
\hline Control (unweeded) & $\begin{array}{c}4.51 \\
(19.34)\end{array}$ & $\begin{array}{c}7.46 \\
(54.70)\end{array}$ & $\begin{array}{c}6.36 \\
(39.55)\end{array}$ & $\begin{array}{c}5.31 \\
(27.24)\end{array}$ & $\begin{array}{c}6.69 \\
(43.08)\end{array}$ & $\begin{array}{c}5.18 \\
(25.88)\end{array}$ \\
\hline Polymulch of $0.25 \mathrm{~mm}$ thickness & $\begin{array}{c}2.20 \\
(3.93)\end{array}$ & $\begin{array}{c}3.03 \\
(8.30)\end{array}$ & $\begin{array}{c}3.39 \\
(10.55)\end{array}$ & $\begin{array}{c}2.75 \\
(6.56)\end{array}$ & $\begin{array}{c}4.56 \\
(19.86)\end{array}$ & $\begin{array}{c}4.04 \\
(15.31)\end{array}$ \\
\hline $\mathrm{SE}(\mathrm{m}) \pm$ & 0.18 & 0.15 & 0.13 & 0.09 & 0.09 & 0.06 \\
\hline C.D. $(p=0.05)$ & 0.55 & 0.44 & 0.41 & 0.29 & 0.29 & 0.20 \\
\hline
\end{tabular}

Figures in parenthesis are original values and data is subjected $\sqrt{X+1}$ transformation 
Table.3 Weed Control Efficiency (\%) and Weed Index (\%) as influenced by weed control options adopted in cotton

\begin{tabular}{|c|c|c|c|c|c|c|c|c|}
\hline \multirow[t]{3}{*}{ Treatments } & \multicolumn{6}{|c|}{ Weed Control Efficiency (\%) } & \multicolumn{2}{|c|}{ Weed Index $(\%)$} \\
\hline & \multicolumn{3}{|c|}{ Red soil } & \multicolumn{3}{|c|}{ Black soil } & & \\
\hline & $30 \mathrm{DAS}$ & 60 DAS & 90 DAS & $30 \mathrm{DAS}$ & 60 DAS & 90 DAS & Red soil & $\begin{array}{c}\text { Black } \\
\text { soil }\end{array}$ \\
\hline $\begin{array}{l}\text { Diuron } 80 \% \text { WP } 0.5 \mathrm{~kg} \mathrm{ha}^{-1} \text { fb pyrithiobac sodium } \\
10 \% \text { EC } 62.5 \mathrm{~g} \mathrm{ha}^{-1}+\text { quizalofop p ethyl } 5 \% \text { EC } 50 \mathrm{~g} \\
\text { ha }^{-1}\end{array}$ & 53.86 & 42.68 & 35.49 & 61.78 & 41.39 & 41.25 & 33.14 & 43.54 \\
\hline $\begin{array}{l}\text { Diuron } 80 \% \text { WP } 0.75 \mathrm{~kg} \mathrm{ha}^{-1} \text { fb pyrithiobac sodium } \\
10 \% \text { EC } 62.5 \mathrm{~g} \mathrm{ha}^{-1}+\text { quizalofop p ethyl } 5 \% \text { EC } 50 \mathrm{~g} \\
\text { ha }^{-1}\end{array}$ & 76.19 & 53.49 & 54.85 & 88.89 & 72.34 & 65.23 & 23.06 & 3.43 \\
\hline $\begin{array}{l}\text { Diuron } 80 \% \text { WP } 1.0 \mathrm{~kg} \mathrm{ha}^{-1} \text { fb pyrithiobac sodium } \\
10 \% \text { EC } 62.5 \mathrm{~g} \mathrm{ha}^{-1}+\text { quizalofop p ethyl } 5 \% \text { EC } 50 \mathrm{~g} \\
\mathrm{ha}^{-1}\end{array}$ & 79.49 & 67.79 & 62.40 & 91.40 & 77.27 & 66.06 & 2.93 & 25.79 \\
\hline $\begin{array}{l}\text { Pendimethalin } 38.7 \% \mathrm{CS} \text { at } 677 \mathrm{~g} \mathrm{ha}^{-1} \text { fb pyrithiobac } \\
\text { sodium } 10 \% \text { EC } 62.5 \mathrm{~g} \mathrm{ha}^{-1}+\text { quizalofop p ethyl } 5 \% \\
\text { EC } 50 \mathrm{~g} \mathrm{ha}^{-1}\end{array}$ & 52.67 & 40.03 & 38.18 & 62.50 & 38.00 & 31.96 & 34.12 & 45.52 \\
\hline $\begin{array}{l}\text { Cotton + sunhemp (sunhemp was cut and spread as } \\
\text { mulch) }\end{array}$ & 34.17 & 28.88 & 31.62 & 48.31 & 55.37 & 26.14 & 69.26 & 62.46 \\
\hline Mechanical weeding at 20, 40, 60 DAS (weed free) & 88.95 & 86.08 & 75.29 & 94.86 & 82.88 & 65.12 & 0.00 & 0.00 \\
\hline Control (unweeded) & 0.00 & 0.00 & 0.00 & 0 & 0 & 0 & 96.05 & 96.30 \\
\hline Polymulch of $0.25 \mathrm{~mm}$ thickness & 85.79 & 84.93 & 73.50 & 94.65 & 79.77 & 64.25 & -15.02 & -26.01 \\
\hline
\end{tabular}


Table.4 Yield and Harvest Index as influenced by weed management practices adopted

\begin{tabular}{|c|c|c|c|c|c|c|}
\hline \multirow[t]{3}{*}{ Treatments } & \multicolumn{3}{|c|}{ Red soil } & \multicolumn{3}{|c|}{ Black soil } \\
\hline & \multicolumn{2}{|c|}{ Yield $\left(\mathrm{Kg} \mathrm{ha}^{-1}\right)$} & \multirow[t]{2}{*}{$\mathrm{HI}$} & \multicolumn{2}{|c|}{ Yield $\left(\mathrm{Kg} \mathrm{ha}^{-1}\right)$} & \multirow[t]{2}{*}{ HI } \\
\hline & Kapas Yield & Stalk Yield & & Kapas Yield & Stalk Yield & \\
\hline $\begin{array}{l}\text { Diuron } 80 \% \text { WP } 0.5 \mathrm{~kg} \mathrm{ha}^{-1} \text { fb pyrithiobac sodium } 10 \% \text { EC } 62.5 \\
\mathrm{~g} \mathrm{ha}^{-1}+\text { quizalofop p ethyl } 5 \% \text { EC } 50 \mathrm{~g} \mathrm{ha}^{-1}\end{array}$ & 1,409 & 3020 & 31.82 & 1,552 & 3074 & 33.55 \\
\hline $\begin{array}{l}\text { Diuron } 80 \% \text { WP } 0.75 \mathrm{~kg} \mathrm{ha}^{-1} \text { fb pyrithiobac sodium } 10 \% \mathrm{EC} \\
62.5 \mathrm{~g} \mathrm{ha}^{-1}+\text { quizalofop p ethyl } 5 \% \text { EC } 50 \mathrm{~g} \mathrm{ha}^{-1}\end{array}$ & 1,622 & 3425 & 32.14 & 2,655 & 4447 & 37.38 \\
\hline $\begin{array}{l}\text { Diuron } 80 \% \text { WP } 1.0 \mathrm{~kg} \mathrm{ha}^{-1} \text { fb pyrithiobac sodium } 10 \% \text { EC } 62.5 \\
\mathrm{~g} \mathrm{ha}^{-1}+\text { quizalofop p ethyl } 5 \% \text { EC } 50 \mathrm{~g} \mathrm{ha}^{-1}\end{array}$ & 2,046 & 4285 & 32.56 & 2,040 & 4122 & 33.11 \\
\hline $\begin{array}{l}\text { Pendimethalin } 38.7 \% \text { C.S. at } 677 \mathrm{~g} \mathrm{ha}^{-1} \text { fb pyrithiobac sodium } \\
10 \% \text { EC } 62.5 \mathrm{~g} \mathrm{ha}^{-1}+\text { quizalofop p ethyl } 5 \% \text { EC } 50 \mathrm{~g} \mathrm{ha}^{-1}\end{array}$ & 1,389 & 3911 & 26.21 & 1,498 & 3058 & 32.88 \\
\hline $\begin{array}{l}\text { Cotton + green manure crop (green manure crop will be cut and } \\
\text { spread as mulch) }\end{array}$ & 648 & 2714 & 19.28 & 1,032 & 2355 & 30.47 \\
\hline Mechanical weeding at 20, 40, 60 DAS (weed free) & 2,108 & 4366 & 32.56 & 2,749 & 4573 & 37.55 \\
\hline Control (unweeded) & 83 & 1113 & 6.96 & 102 & 1255 & 7.49 \\
\hline Polymulch of $0.25 \mathrm{~mm}$ thickness & 2,425 & 4908 & 33.07 & 3,464 & 5585 & 38.28 \\
\hline $\mathrm{SE}(\mathbf{m}) \pm$ & 121.6 & 192.9 & & 211.6 & 176.49 & \\
\hline C.D. & 372.4 & 590.77 & & 648.1 & 540.51 & \\
\hline
\end{tabular}


Weed Index as influenced by different weed control treatments in red and black soils is presented in table 3 .

\section{Red soil}

Lowest weed index was recorded in diuron $1.0 \mathrm{~kg} \mathrm{ha}^{-1}$ as PE $f b$ pyrithiobac sodium + quizalofop $\mathrm{p}$ ethyl as PoE (2.93\%). Diuron $0.75 \mathrm{~kg} \mathrm{ha}^{-1}$ as PE $f b$ pyrithiobac sodium + quizalofop p ethyl as PoE also recorded lower weed index (23.06\%).Whereas diuron $0.5 \mathrm{~kg}$ $\mathrm{ha}^{-1}$ as PE $f b$ pyrithiobac sodium + quizalofop $\mathrm{p}$ ethyl as PoE and pendimethalin as PE $f b$ pyrithiobac sodium + quizalofop $\mathrm{p}$ ethyl as PoE recorded higher weed index (33.14 and $34.12 \%$ respectively) compared to higher doses of diuron. Unweeded control and intercropping of cotton with sunhemp recorded higher weed index values indicating the ineffectiveness of the treatment. Weed index was negative (-15.02) in polymulch treatment due to higher yield recorded compared to weed free treatment i.e. mechanical weeding thrice.

\section{Black soil}

Similar to that of red soil the negative weed index was recorded in polymulch treatment ($26.01 \%$ ). Diuron $0.75 \mathrm{~kg} \mathrm{ha}^{-1}$ as PE $f b$ pyrithiobac sodium + quizalofop $\mathrm{p}$ ethyl as POE\% (3.43\%) recorded the lowest weed index indicating the lowest difference of yield with that of weed free treatment and not by diuron $1.0 \mathrm{~kg} \mathrm{ha}^{-1}$ as PE $f b$ pyrithiobac sodium + quizalofop p ethyl as PoE (25.79\%) which may be due to the mortality of plants by the phytotoxicity which resulted in lower plant population thereby lesser yield. And also diuron $0.5 \mathrm{~kg} \mathrm{ha}^{-1}$ and pendimethalin as PE $f b$ sequential application of herbicides also reported higher weed indices $(43.54 \%$ and $45.53 \%$ respectively) than above mentioned treatments. Similar to that of red soil, the intercropping treatment resulted in highest weed index in unweeded control. Similar trend was observed by Kurlekar and Khuspe (1979) who reported that weeding and interculturing treatment gave the efficient control of weeds and also the application of diuron treatment decreased the intensity of both monocot and dicot weeds.

\section{Yield}

\section{Kapas Yield $\left(\mathrm{kg} \mathrm{ha}^{-1}\right)$}

The weed control practices adopted have greatly influenced the kapas yield and the data pertaining to the yield is presented in the table 4.

\section{Red soil}

Among the weed control practices adopted, polymulch $\left(2425 \mathrm{~kg} \quad \mathrm{ha}^{-1}\right)$ recorded significantly highest yield which was on par with mechanical weeding thrice $(2108 \mathrm{~kg}$ ha $\left.{ }^{1}\right)$. Among the herbicides, diuron $1.0 \mathrm{~kg} \mathrm{ha}^{-1}$ PE $f b$ pyrithiobac sodium + quizalofop $\mathrm{p}$ ethyl PoE (2046 kg ha $\left.{ }^{-1}\right)$ was statistically comparable to mechanical weeding and significantly superior to the other chemical treatments. Diuron at $0.75 \mathrm{~kg} \mathrm{ha}^{-1} \mathrm{PE} f b$ pyrithiobac sodium + quizalofop $\mathrm{p}$ ethyl $\mathrm{PoE}$ was comparable to diuron $0.5 \mathrm{~kg} \mathrm{ha}^{-1}$ and pendimethalin $\mathrm{PE} f b$ pyrithiobac sodium + quizalofop $\mathrm{p}$ ethyl PoE. The unweeded control (83 kg ha 1 ) registered the lowest yield.

Polymulch, mechanical weeding thrice and diuron $1.0 \mathrm{~kg} \mathrm{ha}^{-1} \mathrm{PE} f b$ pyrithiobac sodium + quizalofop $\mathrm{p}$ ethyl $\mathrm{PoE}$ recorded $96.57 \%$, $96.06 \%$ and $95.94 \%$ respectively higher yield compared to unweeded control.

\section{Black soil}

Polymulch (3464 kg ha ${ }^{-1}$ ) recorded the highest yield and was significantly superior to 
the mechanical weeding thrice $\left(2749 \mathrm{~kg} \mathrm{ha}^{-1}\right)$. Unlike the red soils, the diuron $0.75 \mathrm{~kg} \mathrm{ha}^{-1}$ PE $f b$ pyrithiobac sodium + quizalofop $\mathrm{p}$ ethyl PoE (2655 kg ha ${ }^{-1}$ ) recorded the highest yield among the chemical weed control practices and was comparable to mechanical weeding treatment.

While the higher dose of $1.0 \mathrm{~kg} \mathrm{ha}^{-1}$ of diuron did not result in higher yields due to the reduced plant stand by phytotoxic effect of the chemical. Diuron $80 \%$ WP at $0.5 \mathrm{~kg}^{-1}$ as $\mathrm{PE} f b$ pyrithiobac sodium + quizalofop $\mathrm{p}$ ethyl as PoE, pendimethalin as $\mathrm{PE} f b$ pyrithiobac sodium + quizalofop $\mathrm{p}$ ethyl as PoE and inter crop with sunhemp were comparable in terms of yield. Even in black soils also the unweeded control (102 kg ha $\left.{ }^{-1}\right)$ recorded the lowest yield.

Unweeded control registered 97.57\%, 96.28\% and $96.16 \%$ reduction in yield compared to polymulching, mechanical weeding and diuron $0.75 \mathrm{~kg} \mathrm{ha}^{-1} \mathrm{PE} f b$ pyrithiobac sodium + quizalofop p ethyl POE respectively.

In both the soils the polymulch treatment achieved the highest yield due to the luxuriant growth of the crop due to the conservation of moisture thereby greater availability of nutrients along with reduced weed competition. Optimum soil moisture results in good plant growth, development and subsequently higher yield. More soil moisture percentage under mulch treatments was due to less evaporational water loss and less number of weeds. As mulch provides favourable condition for plant growth so higher number and bolls were recorded in mulched treatment as compared to unmulch treatment. These results are in accordance with the findings of Nasrullah et al., (2011).

Lower kapas yield with diuron at $1.0 \mathrm{~kg} \mathrm{ha}^{-1}$ in black soil which was mainly due to reduction in plant population due to phytotoxic effect of diuron during early stage of crop growth which was indicated by yellowing, chlorosis, wilting, and swelling of roots without root hairs and finally death of seedling was observed. This confirms results of Hanumanth (2017).

Herbicidal treatments recorded higher kapas yield which could be due to the enhanced plant height, dry matter production and nutrient uptake of the crop. This might also be due to the season long weed control which was favourable for better growth and enhanced leaf area contributing for the activated photosynthesis and translocation of more photosynthates to sink which increased the yield. These results are in accordance with Nalini et al., (2011) and Prabhu (2011).

\section{Stalk yield ( $\mathrm{kg} \mathrm{ha}^{-1}$ )}

Stalk yield also was significantly influenced by the weed control methods and the data is presented in table 4 .

\section{Red soil}

The perusal of data on stalk yield indicated that polymulch (4908 $\left.\mathrm{kg} \mathrm{ha}^{-1}\right)$ and mechanical weeding thrice (4366 $\mathrm{kg} \mathrm{ha}^{-1}$ ) recorded the significantly highest stalk yield. Diuron 1.0 $\mathrm{kg} \mathrm{ha}^{-1} \mathrm{PE} f b$ pyrithiobac sodium + quizalofop p ethyl as PoE (4258 $\mathrm{kg} \mathrm{ha}^{-1}$ ) was on par with mechanical weeding. Among the herbicides, diuron $0.75 \mathrm{~kg} \mathrm{ha}^{-1}$, diuron $0.5 \mathrm{~kg} \mathrm{ha}^{-1}$ and pendimethalin as PE $f b$ pyrithiobac sodium + quizalofop $p$ ethyl as PoE were on par statistically. The unweeded control $(1113 \mathrm{~kg}$ $\mathrm{ha}^{-1}$ ) registered the lowest stalk yield.

\section{Black soil}

Polymulch $\quad\left(5585 \quad \mathrm{~kg} \quad \mathrm{ha}^{-1}\right) \quad$ recorded significantly greater stalk yield compared to the other treatments. Mechanical weeding thrice (4573 $\mathrm{kg} \mathrm{ha}^{-1}$ ), diuron $0.75 \mathrm{~kg} \mathrm{ha}^{-1}$ 
(4474 $\left.\mathrm{kg} \mathrm{ha}^{-1}\right)$ and diuron $1.0 \mathrm{~kg} \mathrm{ha}^{-1}(4122 \mathrm{~kg}$ $\left.\mathrm{ha}^{-1}\right)$ as PE $f b$ pyrithiobac sodium + quizalofop $p$ ethyl PoE were comparable to each other were the next best treatments. Even in black soils also the unweeded control $\left(1255 \mathrm{~kg} \mathrm{ha}^{-1}\right)$ recorded the lowest yield.

However in both the soils the polymulch treatment achieved the highest stalk yield due to the luxuriant vegetative growth of the crop due to the conservation of moisture thereby greater availability of nutrients along with reduced weed competition. While the higher dose of $1.0 \mathrm{~kg} \mathrm{ha}^{-1}$ of diuron did not record in higher stalk yields due to the reduced plant stand by phytotoxic effect of the chemical.

\section{Harvest index (HI)}

The data regarding harvest index was presented in the table 4 .

\section{Red soil}

Polymulch (33.07) registered superior HI which was followed by mechanical weeding thrice (32.56). Among the chemical treatments diuron $1.0 \mathrm{~kg} \mathrm{ha}^{-1}$ (32.56) and 0.75 $\mathrm{kg} \mathrm{ha}^{-1}$ (32.14) $\mathrm{fb}$ pyrithiobac sodium + quizalofop p ethyl as PoE recorded greater HI than $0.5 \mathrm{~kg} \mathrm{ha}^{-1}$ and pendimethalin treatments which were $f b$ intercrop with sunhemp. The least HI was noticed in unweeded control (6.96).

\section{Black soil}

The persual of data on $\mathrm{HI}$ indicated that polymulch (38.28) recorded superior HI which was followed by mechanical weeding thrice (37.55). Among the chemical treatments $0.75 \mathrm{~kg} \mathrm{ha}^{-1}$ (37.38) $f b$ pyrithiobac sodium + quizalofop p ethyl as PoE recorded greater $\mathrm{HI}$ than diuron $1.0 \mathrm{~kg} \mathrm{ha}^{-1}, 0.5 \mathrm{~kg} \mathrm{ha}^{-1}$ and pendimethalin treatments which were $f b$ intercrop with sunhemp. The least HI was noticed in unweeded control (7.49). Similar results were reported by Patil et al., (1998), Panwar et al., (2001).

\section{References}

Ather, M. N., Idrees, N. M., Ayub, M., Tanveer, A and Mubeen, K. 2013. Effect of different weed control practices and sowing methods on weeds and yield of cotton. Pakistan Journal of Botany. 45: 1321-1328.

Chetan, K.S., Salakinkop, S.R., Angadi, S.S. 2016. Economic viability of sequential application of pre and post emergence herbicides in Bt cotton. Journal of Cotton Research and Development. 30(1): 79-83.

Hanumanth, M. 2017. Productivity, profitability and environmental impact of different weed management options in high density Cotton (Gossypium hirsutum L.). Msc thesis. Professor Jayashankar Telangana State Agricultural University. Rajendranagar, Hyderabad.

Hargilas, Ameta, G.S., Jat, S. C and Saini, D.P. 2015. Evaluation of effective weed management strategy for $\mathrm{BT}$ cotton. The Bioscan. 10(3): 1313-1316.

Hariharasudhan, V., Chinnusamy, C and Prabhakaran, N.K. 2017. Optimum time of sowing and weed management methods on weeds interference and productivity and profitability of $B t$ cotton hybrid in western zone of Tamil Nadu. International Journal of Chemical Studies. 5(3): 793-796.

Hiremath, R.., Yadahalli, G.S., Chittapur, B.M., Siddapur, A.D., Yadahalli, V.G. and Koppalkar, B.R.G.2013. Integrated weed management in Bt. Cotton (Gossypium hirsutum L.) under UKP command area of Karnataka. Acta Biologica Indica. 2 (2): 400-405. 
Kurlekar, V.G and Khuspe, V.S. 1979. Studies on weed control in cotton (H-4). Indian Journal of Agronomy. 24(4): 377-382.

Nalini, K., Muthukrishan, P., Chinnusamy, C and Janaki, P. 2013. Response of soil microflora in herbicide residue of winter irrigated cotton. Crop Research. 45(1/3): 268-271.

Nalini, K., Muthukrishnan, $\mathrm{R}$ and Chinnusamy, C. 2011. Evaluation of Pendimethalin 38.7 EC on Weed Management in Winter Irrigated Cotton. Madras Agricultural Journal. 98(4-6): 165-168.

Nasrullah, M., Khan, M. B., Ahmad, R., Ahmad, S., Hanif, $M$ and Nazeer, W. 2011. Sustainable cotton production and water economy through different planting methods and mulching techniques. Pakistan Journal of Botany. 43: 1971-1983.

Panwar, R.S., Balyan, R.S and Malik, R.K. 2001. Evaluation of gluphosinate for control of weeds in cotton. Indian
Journal of Weed Sciences. 32 (1\&2): 94-95.

Patil, B.M., Satao R.N and Lohariya, G.S. 1998. Integrated weed management in cotton. PKV-Research Journal. 21 (2): 220-221.

Prabhu G, Halepyati A, Pujari B.T and Desai B.K. 2011. Integrated weed management in $\mathrm{Bt}$ cotton (Gossypium hirsutum L.) under irrigated conditions. Karnataka Journal of Agricultural Sciences. 24(4): 529- 530.

Prabhu, M. 2010. Evaluation of integrated weed management practices in $B t$ cotton. M.Sc., Thesis, Tamil Nadu Agricultural University, Coimbatore, Tamil Nadu, India, p.112.

Sharma, R. 2008. Integrated weed management in field crops. Crop Care. 35 (4): 41-46.

Shelke, D.K. and Bhosle, R.H., 1990, Determination of critical period of cropweed competition in rainfed cotton. Journal of Maharashtra Agricultural Universities. 15(2): 257-258.

\section{How to cite this article:}

Varsha N., M. Madhavi, T. Ramprakash and Suneetha Devi K. B. 2018. Efficacy of Diuron Along with Sequential Application of Herbicides for Weed Control in Cotton. Int.J.Curr.Microbiol.App.Sci. 7(06): 3423-3436. doi: https://doi.org/10.20546/ijcmas.2018.706.401 